\title{
Of Amoebae and Men: Extracellular DNA Traps as an Ancient Cell-Intrinsic Defense Mechanism
}

\author{
Xuezhi Zhang and Thierry Soldati* \\ Department of Biochemistry, Science II, University of Geneva, Geneva, Switzerland
}

Since the discovery of the formation of DNA-based extracellular traps (ETs) by neutrophils as an innate immune defense mechanism (1), hundreds of articles describe the involvement of ETs in physiological and pathological human and animal conditions [reviewed in Ref. (2), and the previous Frontiers Research Topic on NETosis: http://www. frontiersin.org/books/NETosis_At_the_Intersection_of_Cell_Biology_Microbiology_and_ Immunology/195]. Interestingly, a few reports reveal that ETs can be formed by immune cells of more ancient organisms, as far back as the common ancestor of vertebrates and invertebrates (3). Recently, we reported that the Sentinel cells of the multicellular slug of the social amoeba Dictyostelium discoideum also produce ETs to trap and kill slug-invading bacteria [see Box 1; and Figure 1 Ref. (4)]. This is a strong evidence

OPEN ACCESS

Edited by:

Marko Radic,

University of Tennessee, USA

Reviewed by:

Magdalena Chadzińska, Jagiellonian University, Poland

Borko Amulic,

Max Planck Institute, Germany

*Correspondence:

Thierry Soldati

thierry.soldati@unige.ch

Specialty section:

This article was submitted to Molecular Innate Immunity, a section of the journal

Frontiers in Immunology

Received: 30 April 2016 Accepted: 27 June 2016 Published: 08 July 2016

Citation:

Zhang $X$ and Soldati T (2016) Of Amoebae and Men: Extracellular DNA Traps as an Ancient CellIntrinsic Defense Mechanism.

Front. Immunol. 7:269. doi: 10.3389/fimmu.2016.00269 that DNA-based cell-intrinsic defense mechanisms emerged much earlier than thought, about 1.3 billion years ago. Amazingly, using extrusion of DNA as a weapon to capture and kill uningestable microbes has its rationale. During the emergence of multicellularity, a primitive innate immune system developed in the form of a dedicated set of specialized phagocytic cells. This professionalization of immunity allowed the evolution of sophisticated defense mechanisms including the sacrifice of a small set of cells by a mechanism related to NETosis. This altruistic behavior likely emerged in steps, starting from the release of "dispensable" mitochondrial DNA by D. discoideum Sentinel cells. Grounded in this realization, one can anticipate that in the near future, many more examples of the invention and fine-tuning of ETs by early metazoan ancestors will be identified. Consequently, it can be expected that this more complete picture of the evolution of ETs will impact our views of the involvement and pathologies linked to ETs in human and animals.

Keywords: amoebozoa, Dictyostelium, NOX, neutrophil extracellular traps, evolution, unicellular eukaryotes, multicellularity, innate immunity

During early evolution of multicellularity, when autonomous eukaryotic single-cell hosts were encountering prokaryotes, they either phagocytosed them as food or moved away to avoid being infected. However, when multicellular organisms evolved, they had to face more directly a serious problem, namely, infection of only parts or tissues of the organism. One solution is what happens in slugs of $D$. discoideum, in which invading bacteria are trapped by patrolling $S$ cells that are subsequently shed behind during slug migration, keeping the multicellular structure free from infection $(4,8)$. The phagocytes in higher animals and men follow similar strategies to circumscribe the infection. For example, patrolling neutrophils catch the invaders and commit suicide, being finally 
discarded by the intervention of macrophages (9). However, in plants, which are immobile and do not have circulating (innate immune) cells, and whose cells have a rigid wall, another strategy had to be co-opted to isolate infected parts from healthy tissues. One such solution is the formation of a callus or tumor induced by wounding and pathogen infections (10). Naturally, a logical question is whether extracellular DNA is also involved in plant callus and tumor formation, a topic unaddressed yet, but that might reveal interesting aspects of the evolution of innate immune defenses in the broadest way.

Dictyostelium discoideum is a remarkable model organism to study the functions of specific genes involved in the emergence

BOX 1 | Dictyostelium discoideum as a unique model to study evolution of innate immunity.

The social amoeba Dictyostelium discoideum belongs to the Amoebozoa, a sister group to the animals and fungi that branched after the divergence of plants $(5,6)$. The life cycle of $D$. discoideum comprises two major stages, a single-celled amoeboid stage and a "social" facultative multicellular stage. During the former, amoebae feed on bacteria and yeasts by phagocytosis, a biological process extremely well conserved in evolution and essentially shared between protozoan phagocytes and phagocytes of the animal innate immune system (7). These features make this genetically tractable organism a unique model to study the function of specific genes involved in the early evolution of innate immunity and the emergence of multicellularity. of multicellularity and the early evolution of cell autonomous defenses (8). Our recent study revealed that extracellular trap (ET) generation evolved much earlier than the emergence of metazoan and that reactive oxygen species (ROS) generated by NADPH oxidases (NOX) are essential in this conserved process (4). Therefore, in this perspective article, we would like to present the provocative hypothesis that, within some limits, the evolutionary history of ROS-generating NOX enzymes may serve as a general signature, a guiding principle that will be useful for the future discovery and study of ETs in other ancient organisms. At this point and before we develop further our arguments in favor of this causal relationship, we would like the reader to note that NADPH- or ROS-independent pathways may also contribute to ET formation under specific stimulations and conditions (11-14), an emerging field that was comprehensively reviewed by Stoiber et al. (15). Although different groups of organisms may employ different molecular machineries to finetune the production of ETs, and the sources of DNA may also vary depending on the process involved $(1,2,4,16,17)$, but from a conceptual point of view, the strategies are similar, using DNA as a weapon for host defense.

Nevertheless, based on our previous phylogenetic investigations, ROS-generating NOX enzymes evolved from metal reducing ferric reductases (FRE) through a functional shift (18). As shown in Table 1, one could speculate that the organisms

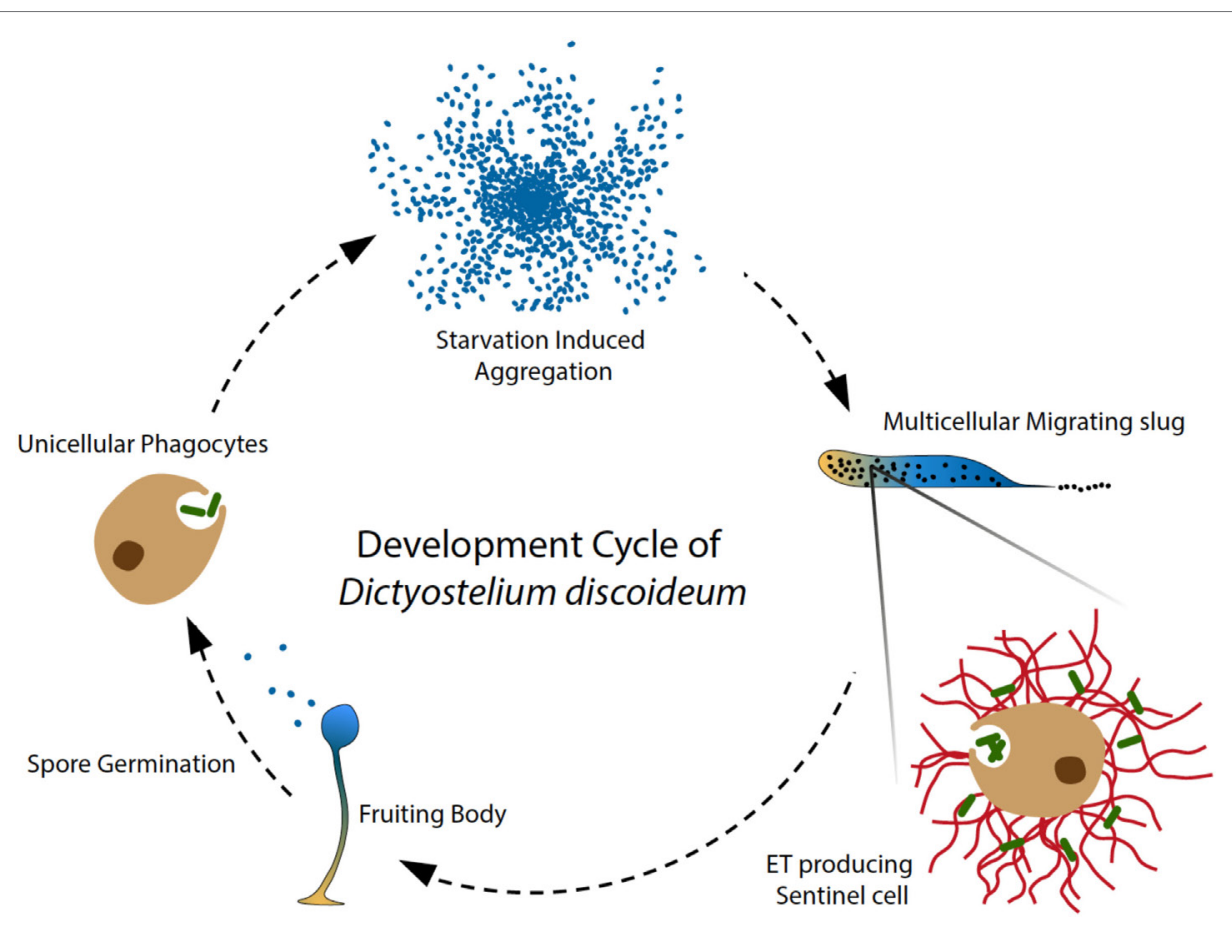

FIGURE 1 | Amoeba phagocytes and Sentinel cells capture and kill bacteria. In the soil, solitary D. discoideum cells feed on bacteria, and starvation induces a developmental program, in which around 100,000 amoebae aggregate to form a migrating multicellular slug, followed by terminal differentiation and generation of fruiting bodies (28). During the migrating slug stage, only a few specialized cells, namely Sentinel (S) cells, keep their original phagocytic capacity and circulate through the slug to capture and kill invading microbes, functioning as a primitive innate immune system at the emergence of multicellular organism (8). In addition, the phagocytic $S$ cells are constantly generated and sloughed off as the slug migrates. Our recent discovery showed that the $S$ cells in the migrating slug of $D$. discoideum can produce extracellular DNA traps in a process that depends on production of reactive oxygen species (ROS) by NOX enzymes. Interestingly, S cells appear to mainly use their mitochondrial DNA to build up ETs, dissociating trap formation from immediate cells death by NETosis. Our study revealed that ET formation is a widespread DNA-based host defense strategy that may have been present in the ancestor of metazoa and amoebozoa. 
TABLE 1 | The co-emergence of NOX enzymes and multicellularity might also correlate with the origin of DNA-based defense strategies.

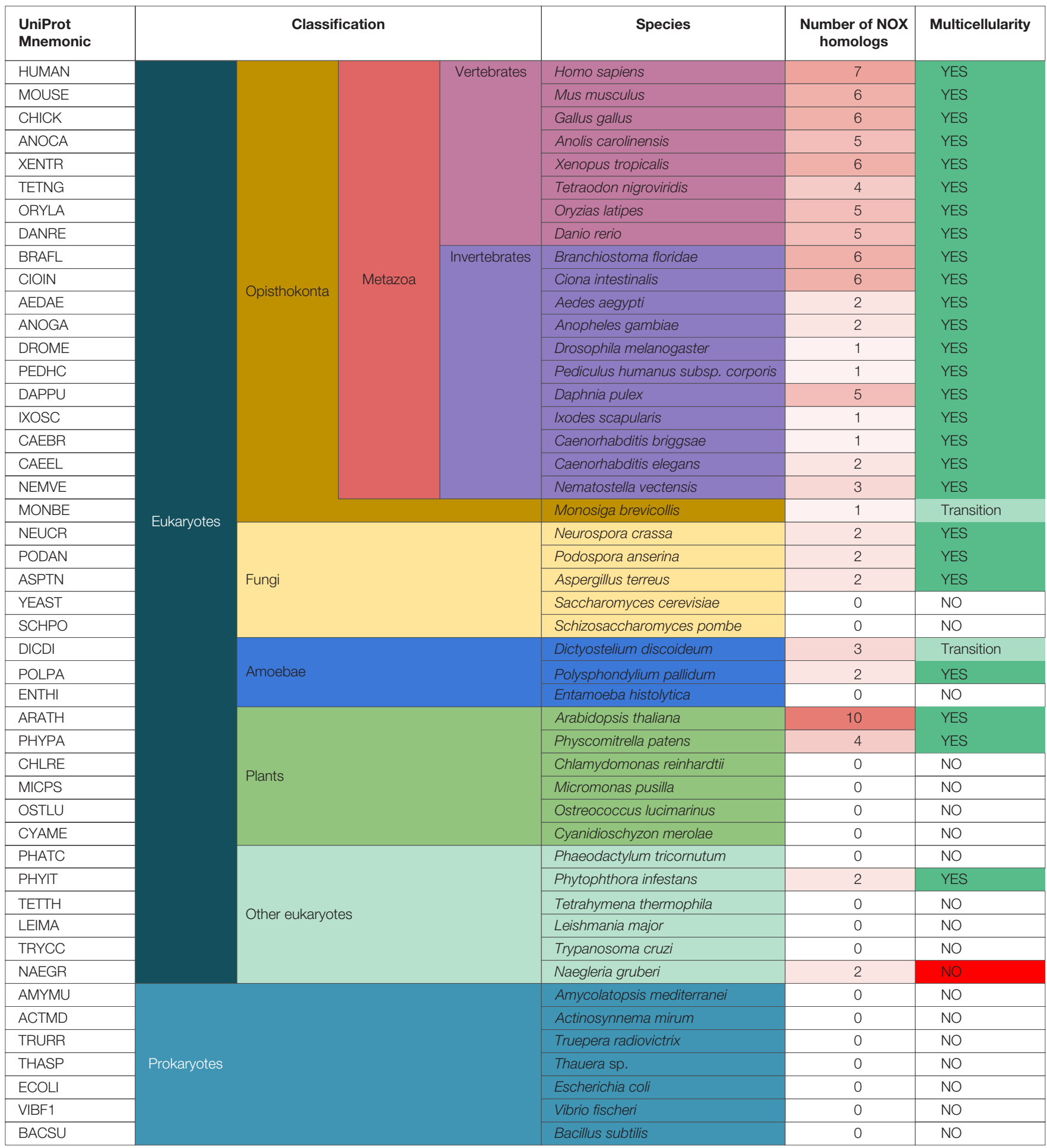

Representative organisms from both eukaryotes and prokaryotes [see Ref. (18) for detailed presentation] were collected and organized by major branches in taxonomy. The number of NOX enzymes in each organism is indicated and color coded. Unicellular and multicellular organisms are indicated by a "NO" and "YES," respectively. Two organisms that are at the transition between the two life forms or have both life forms are indicated as "Transition." One exception is Naegleria gruberi, a single-celled organism well known for its capacity to transition from an amoeboid to a flagellated form. It is a free-living organism, but closely related to pathogenic, parasitic species. Therefore, it is plausible that the NOX gene of Naegleria might have been acquired from its host via horizontal gene transfer or that it derives from an organism that was at the transition to multicellularity, but lost this characteristic of multicellular organisms as it specialized to its environment. The discovery of NOX-dependent ET generation in the multicellular form of the amoeba D. discoideum, an organism that is at the transition to multicellularity, combined to the recognition of the apparent coemergence of multicellularity and NOX enzymes indicate that the origin of ET formation might be traced back to the emergence of multicellular organisms. It also suggests that variants and diverse evolutions of DNA-based defense strategies might be identified in other organisms with functional NOX enzymes, both in primitive metazoans and organisms close to the transition to multicellularity. 
that express NOX homologs can generate ROS as signaling molecules to trigger ET formation for host defense. Interestingly, the evolutionary time of emergence of experimentally confirmed ET formation, NOX function, and multicellularity coincide well, possibly indicating that ROS-dependent DNA-based host defenses played a critical role in the early evolution of multicellular organisms guarded by an innate immune system.

In the near future, DNA-based host defense strategies will certainly be identified in a growing number of organisms. We propose that their study will reveal the fundamental significance in the relationship between host organisms and their coexisting commensals and pathogens and bring conceptual changes in the way we approach many relevant human diseases. For example, in higher plants, the roots have direct contact with various microbes in the soil, and among the various host defense mechanisms, the root border cells are able to secrete extracellular DNA to trap and kill bacteria and fungi $(19,20)$. In analogy, the human gut is colonized by large numbers of microbes, collectively referred to as the microbiota (21). While maintaining intimate contact with the normal microbiota, the intestinal epithelial cells are at the front of host-microbe interactions (22). Enteric pathogenic bacterial infection and antibiotic treatment are able to dramatically change the metabolic profile of the human microbiota and the gut ecosystem, sometimes leading to systemic inflammation and autoimmune responses. Importantly, unregulated ET formation is a major inducing factor of systemic inflammation and autoimmune

\section{REFERENCES}

1. Brinkmann V, Reichard U, Goosmann C, Fauler B, Uhlemann Y, Weiss DS, et al. Neutrophil extracellular traps kill bacteria. Science (2004) 303:1532-5. doi:10.1126/science. 1092385

2. Goldmann O, Medina E. The expanding world of extracellular traps: not only neutrophils but much more. Front Immunol (2012) 3:420. doi:10.3389/ fimmu.2012.00420

3. Robb CT, Dyrynda EA, Gray RD, Rossi AG, Smith VJ. Invertebrate extracellular phagocyte traps show that chromatin is an ancient defence weapon. Nat Commun (2014) 5:4627. doi:10.1038/ncomms5627

4. Zhang X, Zhuchenko O, Kuspa A, Soldati T. Social amoebae trap and kill bacteria by casting DNA nets. Nat Commun (2016) 7:10938. doi:10.1038/ ncomms 10938

5. Eichinger L, Pachebat JA, Glockner G, Rajandream MA, Sucgang R, Berriman M, et al. The genome of the social amoeba Dictyostelium discoideum. Nature (2005) 435:43-57. doi:10.1038/nature03481

6. Cosson P, Soldati T. Eat, kill or die: when amoeba meets bacteria. Curr Opin Microbiol (2008) 11:271-6. doi:10.1016/j.mib.2008.05.005

7. Boulais J, Trost M, Landry CR, Dieckmann R, Levy ED, Soldati T, et al. Molecular characterization of the evolution of phagosomes. Mol Syst Biol (2010) 6:423. doi:10.1038/msb.2010.80

8. Chen G, Zhuchenko O, Kuspa A. Immune-like phagocyte activity in the social amoeba. Science (2007) 317:678-81. doi:10.1126/science.1143991

9. McCracken JM, Allen LA. Regulation of human neutrophil apoptosis and lifespan in health and disease. J Cell Death (2014) 7:15-23. doi:10.4137/JCD. S11038

10. Ikeuchi M, Sugimoto K, Iwase A. Plant callus: mechanisms of induction and repression. Plant Cell (2013) 25:3159-73. doi:10.1105/tpc.113.116053

11. Parker H, Dragunow M, Hampton MB, Kettle AJ, Winterbourn CC. Requirements for NADPH oxidase and myeloperoxidase in neutrophil extracellular trap formation differ depending on the stimulus. J Leukoc Biol (2012) 92:841-9. doi:10.1189/jlb.1211601 diseases in human (23-27). Therefore, understanding whether intestinal macrophages or neutrophils, or some other intestinal cell types, are able to excrete DNA during enteric pathogenic bacterial infections or antibiotic treatment is an interesting but underexplored area. In the future, understanding the chemical dialogs between gut microbiota and ET formation could potentially lead to new therapies to control and cure these diseases.

In conclusion, formation of ETs is an ancient cell-intrinsic defense mechanism that might have played a critical role in the evolution of multicellular organisms, and we need more systematic approaches and a broader perspective to recognize the importance of ETs in host-commensal and host-pathogen interactions. We expect that more related studies in the future will keep up the excitement in this field of research.

\section{AUTHOR CONTRIBUTIONS}

$\mathrm{XZ}$ and TS designed the experiments and interpreted the results. $\mathrm{XZ}$ performed the experiments and $\mathrm{XZ}$ and $\mathrm{TS}$ wrote the manuscript.

\section{FUNDING}

The work in the TSlab was supported by the European Cooperation in Science and Technology (COST) Action BM1203/EU-ROS, the Swiss SEFRI-COST No. C13.0137, and multiple grants from the Swiss National Science Foundation.

12. Pilsczek FH, Salina D, Poon KK, Fahey C, Yipp BG, Sibley CD, et al. A novel mechanism of rapid nuclear neutrophil extracellular trap formation in response to Staphylococcus aureus. JImmunol (2010) 185:7413-25. doi:10.4049/jimmunol.1000675

13. Nishinaka Y, Arai T, Adachi S, Takaori-Kondo A, Yamashita K. Singlet oxygen is essential for neutrophil extracellular trap formation. Biochem Biophys Res Commun (2011) 413:75-9. doi:10.1016/j.bbrc.2011.08.052

14. Douda DN, Khan MA, Grasemann H, Palaniyar N. SK3 channel and mitochondrial ROS mediate NADPH oxidase-independent NETosis induced by calcium influx. Proc Natl Acad Sci U S A (2015) 112:2817-22. doi:10.1073/ pnas. 1414055112

15. Stoiber W, Obermayer A, Steinbacher P, Krautgartner WD. The role of reactive oxygen species (ROS) in the formation of extracellular traps (ETs) in humans. Biomolecules (2015) 5:702-23. doi:10.3390/biom5020702

16. Yousefi S, Mihalache C, Kozlowski E, Schmid I, Simon HU. Viable neutrophils release mitochondrial DNA to form neutrophil extracellular traps. Cell Death Differ (2009) 16:1438-44. doi:10.1038/cdd.2009.96

17. Yousefi S, Gold JA, Andina N, Lee JJ, Kelly AM, Kozlowski E, et al. Catapultlike release of mitochondrial DNA by eosinophils contributes to antibacterial defense. Nat Med (2008) 14:949-53. doi:10.1038/nm.1855

18. Zhang X, Krause K-H, Xenarios I, Soldati T, Boeckmann B. Evolution of the ferric reductase domain (FRD) superfamily: modularity, functional diversification, and signature motifs. PLoS One (2013) 8:e58126. doi:10.1371/journal. pone. 0058126

19. Hawes MC, Curlango-Rivera G, Wen F, White GJ, Vanetten HD, Xiong Z. Extracellular DNA: the tip of root defenses? Plant Sci (2011) 180:741-5. doi:10.1016/j.plantsci.2011.02.007

20. Driouich A, Follet-Gueye ML, Vicre-Gibouin M, Hawes M. Root border cells and secretions as critical elements in plant host defense. Curr Opin Plant Biol (2013) 16:489-95. doi:10.1016/j.pbi.2013.06.010

21. Lozupone CA, Stombaugh JI, Gordon JI, Jansson JK, Knight R. Diversity, stability and resilience of the human gut microbiota. Nature (2012) 489:220-30. doi: $10.1038 /$ nature 11550 
22. Rescigno $M$. The intestinal epithelial barrier in the control of homeostasis and immunity. Trends Immunol (2011) 32:256-64. doi:10.1016/j.it.2011.04.003

23. Thammavongsa V, Missiakas DM, Schneewind O. Staphylococcus aureus degrades neutrophil extracellular traps to promote immune cell death. Science (2013) 342:863-6. doi:10.1126/science.1242255

24. Yipp BG, Petri B, Salina D, Jenne CN, Scott BN, Zbytnuik LD, et al. Infectioninduced NETosis is a dynamic process involving neutrophil multitasking in vivo. Nat Med (2012) 18:1386-93. doi:10.1038/nm.2847

25. Yost CC, Cody MJ, Harris ES, Thornton NL, McInturff AM, Martinez ML, et al. Impaired neutrophil extracellular trap (NET) formation: a novel innate immune deficiency of human neonates. Blood (2009) 113:6419-27. doi:10.1182/blood-2008-07-171629

26. Fuchs TA, Brill A, Duerschmied D, Schatzberg D, Monestier M, Myers DD Jr, et al. Extracellular DNA traps promote thrombosis. Proc Natl Acad Sci U S A (2010) 107:15880-5. doi:10.1073/pnas.1005743107
27. Bosch X. Systemic lupus erythematosus and the neutrophil. N Engl J Med (2011) 365:758-60. doi:10.1056/NEJMcibr1107085

28. Chisholm RL, Firtel RA. Insights into morphogenesis from a simple developmental system. Nat Rev Mol Cell Biol (2004) 5:531-41. doi:10.1038/nrm1427

Conflict of Interest Statement: The authors declare that the research was conducted in the absence of any commercial or financial relationships that could be construed as a potential conflict of interest.

Copyright $\odot 2016$ Zhang and Soldati. This is an open-access article distributed under the terms of the Creative Commons Attribution License (CC BY). The use, distribution or reproduction in other forums is permitted, provided the original author(s) or licensor are credited and that the original publication in this journal is cited, in accordance with accepted academic practice. No use, distribution or reproduction is permitted which does not comply with these terms. 